\title{
Sur la notion de l'ordre dans la Théorie des Ensembles.
}

\author{
Par \\ Casimir Kuratowski (Varsovie).
}

Les considérations qui sont exposées dans cette Note suivent la voie des idées des MM. Hessenberg ${ }^{1}$ ) et Hartogs') sur la méthode d'introduction de la notion d'ordre dans la Théorie des Ensembles.

Cette méthode peut être resumée, comme suit.

Soit $M$ un ensemble quelconque; convenons de dire que la classe $\left.{ }^{3}\right) \quad \boldsymbol{M}$ "établit un ordre dans l'ensemble $M$ lorsqu'elle vérifie les conditions suivantes:

(1) les éléments de la classe $\boldsymbol{M}$. sont des sous-ensembles de $\boldsymbol{M}$;

(2) $X$ et $Y$ étant des éléments quelconques de $\boldsymbol{M}$ on a:

$$
X \subset Y \text { ou bien } Y \subset \dot{X}
$$

1) Grundbegriffe der Mengenlehre. Abhandlungen der Fries'schen Schule I, 4, Gottingen 1906, p. 674-685 ("Vollstundige ordnende Systeme").

Dans une note pabliée à la même époque (Sur les éléments de la théorie des ensembles ordonnés, Ensoignemont. Mathématique VIII, 1906 Mai-Juin, p. 201) M. Combébiac a exprimé sur la théorie do l'ordre des idées bien analogues a celles do M. Hossen borg.

1) Ueber dais Problem der Wohlordnung. Anbang. Mathomatische Annalen 76, 1914, p. 443.

*) Four la commodité du langago nous ferons usage du terme "classe" lorsqu'il sera question des ensembles, dont los óléments sont eux-mêmes des ensembles par hypothèso; noús désignons les. elasses par $\mathcal{A}, \boldsymbol{B}, \boldsymbol{C}, \ldots$ Leurs élémentsensembles par $A, B \ldots$; los ólérnonte do cós derniers par $a, b \ldots$

$a \& A$

signifie, commo d'habitudo quo a ost un élóment do $A$;

$A \subset B$

signifie que $A$ est un sous-ensemble de $B$, que $A$ est contenu dans $B$.

Fundamenta Malhematicae II. 
(3) $x$ et $y$ étant deux éléments différents do $M$, il existe un ensemble-élément de $\boldsymbol{M}$ qui en contient un suns en contenir l'autre;

(4) $\boldsymbol{X}$ étant une sous-classe de $\boldsymbol{M}$, la somme do tous les ensembles qui sont les éléments de $\boldsymbol{X}$, est un blément de $\boldsymbol{M}$;

(5) il en est de même du produit do cos onsemblos.

On démontre, que lorsque $\boldsymbol{M}$ remplit los conditions $1-5$ et lorsqu'on pose

$$
x<y
$$

quand il existe un élement $Y$ de $\boldsymbol{M}$ dont $y$ est un élóment et $x$ ne l'est pas, - l'ensemble $M$ est ordonné au sent habituel du mot. D'autre part, lorsqu'on suppose que l'onsemblo $M$ est ordonné d'une certaine façon, la classe de tous ses restes ') vérifie les conditions 1-5; elle est d'ailleurs la seule ${ }^{2}$ ) qui les véritio en l'or. donnant de cette façon. Done, il existo une correspondance biunivoque entre les façons d'ordonner un ensemble donné ot los classes qui y nétablissent un ordre".

Ainsi, la thérie des classes qui établissent un ordre peut etre regardée comme équivalente à la théorio elassiquo des ensembles ordonnés, basée sur la notion intuitive d'ordro (Oantor). En même temps, on peut la déduiro do la théorio généralo des

1) On appelle nreste ${ }^{4}$ d'un ensemblo ortonnó toat onsemble qui jouit do la propriété suivanto: lorsque $x$ est son Glément, tont ólómont précélé pur $x$ l'est aussi. (Hesenborg, l, c. p. 5u1).

2) M. Hartoga affirmo, cho $M$ étant un onsemble orlonnó d'uno certnino façon et $\boldsymbol{M}$ uno classe l'ordonnant ainsi ot ne satisfuisant qu'aux conditions $1-4,-M$ est identique a la classe de tous los restes do M. (p. 44.3, lignes: 12-15).

Ceci est inexact. En effet, soit $M$ l'ensomble do toins les $w$ satisfaisunt à l'inégalité: $0 \leqslant x \leqslant 1$; onvisageons la alasse $S$ de tous los segmonts contenus dans $M$ et contenant lo point 1 ; soit $\boldsymbol{M} X_{1}$ lit olusso do tous los onsomblos do points qu'on obtient do cos segmonts on $y$ supprimant los bornes infóriouros. On voit inumédiaternent que $M_{1}$ sutisfait alax conditions 1-4. Soit, d'natro part,

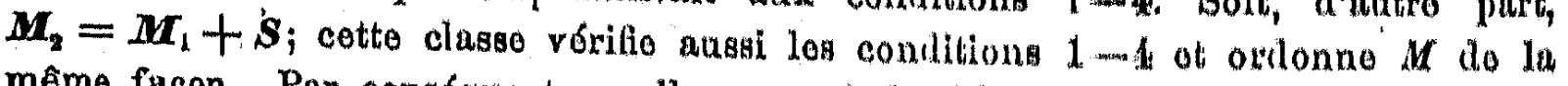
même façon. Par conséquent, quelle quo soitt li dólinition du tormo "rosto", uno au moins de ces deax classos: $\boldsymbol{M}_{1}$ ou $\boldsymbol{M}_{z}$, n'est pus la classo do tonin les restes dé l'ensemble $M$, - contrairoment à l'assertiont da M. Martogs.

Dans cet exemple deax nordxos" différonta (au nons do M. Merartoga) $\boldsymbol{M r}_{1}$ et $\boldsymbol{M}_{2}$ ordonnent d'une soule facon l'onsomble $M$. Cot inconvónient -

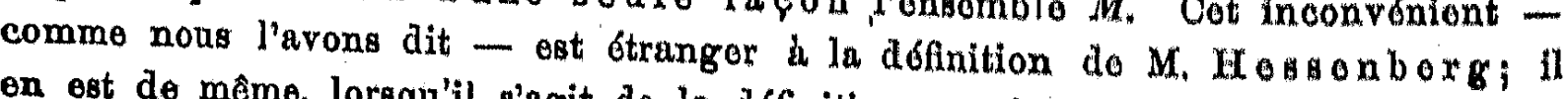
en est de même, lorsqu'il s'agit de la définition que jo propose plua loin. 
ensembles (non ordonnés) sans qu'il y faille introduire aucune notion première supplémentaire: l'idée de l'ordre y est donnée en termes fondamentaux du système des axiomes de M. Zermelo ${ }^{1}$ ), à savoir, celui d'ensemble et celui d'élément ${ }^{2}$ ). L'importance de cette méthode est manifeste.

Nous donnons dans cette note une autre définition de l'ordre, qui nous semble bien naturello et qui équivaut à celle de M. Hessenberg; en outre, nous envisageons la notion de l'ensemble bien ordonné et. celle do la suite; les considérations sur la s u ite finie sont intimement liées a la définition de l'ensemble fini, qui a été proposée dans le volume précédent des „Fundamenta" s).

Solon la terminologie de Janiszews $\mathrm{ki}$ ) un énsemble est dit saturé par rapport à une propriété donnée s'il la possède lui même et s'il n'est un vrai sous-ensemble d'aucun ensemble, qui la possède. D'une façon analogue, un ensemblo est dit irréductible par rapport à une propriété, s'il la possède et si apcun de ses sousensembles ne la possèdo pas.

Ces deux notions ont une grande importance pour plusieurs théories mathématiques. Nous en ferons usage dans cette note.

Convenons de dire, que $\boldsymbol{M}$ est une nclasse d'ensembles décroissants" (ou croissants) lorsqu'il est vrai de tous deux éléments $X$ ot $Y$ do $\boldsymbol{M}$, que

\section{$X \subset Y$ ou bien $Y \subset X$}

En se basant sur cette définition et sur celle de l'ensemble saturé nous introduisons la notion de l'ordre à l'aide de la définition suivante $\left.{ }^{6}\right)$.

1) Untersuchungen Wber die Grunalagen der Mengentehre, Math. Ann. 65, 1908.

*) Uno dófinition d'onsomble ordonné, donnée on termes d'ensemble ot

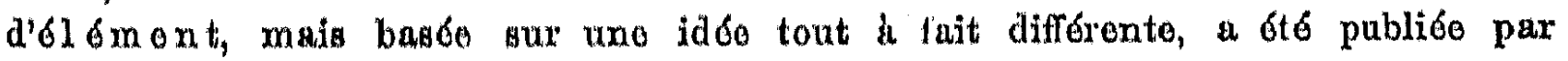
M. Ha a d oxf dans sos Grundallyo der Mengenlehro (Leipuig 1914); voir aussi Kuxatown ki Sur la defmition de la grandeur ${ }_{n} \mathrm{O}$ definieji wiolkosci", Przoglad rilozoflezny, Varsovio, 1817).

4) Kuratow al Sur la notion de l'ensemble fini Varsovio 1920.

4) Janisхow $\mathrm{ki}$ Sur les continus irréductibles entre deux points, p. 7, Paris 1911 (Thèso).

v) Il n'ent question que des onsembles non vides. 
Définition. I. La classe $M$ ordonne l'ensemble $M$, lorsqu'elle est saturée par rapport ì la propriété d'être une classede sous-ensembles décroissants de $M$.

On voit immédiatement que la définition I s'obtient de celle de $M$. Hessenberg en y remplaçant les conditions 3, 4 et 5 par la condition:

(6) $M$ n'est pas une vraie sous-çasse d'aucune classe satisfaisant aux conditions 1 et 2.

Nous allons montrer, que cette définition donne une conception de l'ordre qui équivaut à celle de la théorie classique des ensembles ordonnés.

Démonstration.

1. Supposons que la elasse $M$ satisfait aux conditions 1,2 et 6 et posons

$$
x<. y,
$$

lorsque $x$ et $y$ appartiennent à $M$ et lorsqu'il existe dans $M$ un élément $Y$ tel, que

$$
y \varepsilon Y \text { et } x \text { non }-\varepsilon Y \text {. }
$$

La relation $n<4$ ordonne l'ensemble $M$, c'est à dire, qu'elle vérifie les conditions suivantes:

$(\alpha)$ elle est transitive,

( $\beta$ ) elle est asymmétrique,

$(\gamma)$ elle subsiste entre tous deux éléments de $M$.

En effet, soit $x<y$ et $y<z$; il existe donc deux ensembles $\boldsymbol{Y}$ et $Z$, éléments de $\boldsymbol{M}$, tels que

$$
\begin{array}{ll}
y \varepsilon Y, & x \text { non }-\varepsilon Y \\
z \varepsilon Z, & y \text { non }-\varepsilon Z .
\end{array}
$$

Par conséquent, $Y$ n'est pas contenu dans $Z$; comme, d'autre part, $M$ est une classe d'ensembles décroissants, il en résulte, que $Z$ est contenu dans $Y$. Donc,

$$
z \varepsilon Z \text { et } x \text { non } \varepsilon Z Z \text {, }
$$

ce qui proure que $x<z$. Ainsi, la condition $(\alpha)$ est remplie. Comme la démonstration de $(\beta)$ est immédiate, nous passons a celle de $(\gamma)$.

Soient $a$ et $b$ deux éléments différents de $k l$ et supposons, que 
tout élément $X$ de $\boldsymbol{M}$, contenant $a$, contient en même temps $b$, comme élément; pour établir la condition $(\gamma)$ il suffit de montrer qu'il existe dans $\boldsymbol{M}$ un élément $B$ tel que

$$
b \varepsilon B \text { et } a \text { non }-\varepsilon B \text {. }
$$

Désignons par $P$ le produit (la partie commune) de tous les ensembles appartenant à $\boldsymbol{M}$ et admettant a comme élément; de tels ensembles existent, car en vertu de la condition $6, M$ est en tout cas un élément de $M$. Soit $B$ l'ensemble qu'on obtient de $P$ en y supprimant l'élément $a$. Je dis, que $B$ appartient à $\boldsymbol{M}$.

Soit $X$ un élément quelconque de $\boldsymbol{M}$. Deux cas peuvent se présenter:

10 a $\varepsilon X$; donc, $P \subset X$, d'où:

$$
B \subset X
$$

2. a non- $\varepsilon X$; done, aucun ensemble contenant a n'est pas contenu dans $X$, et - en vertu de la cond. $2-X$ est contenu dans chacun d'eux; il en résulte que $X \subset P$; or, $B$ ne diffère de $P$ que par l'élément $a$ et, par suite,

$$
X \subset B \text {. }
$$

Il est donc vrai de tout $X$ appartenant à $\boldsymbol{M}$, que

$$
B \subset X \text { ou bien } X \subset B
$$

c'est à dire: la classe, admettant comme éléments l'ensemble $B$ et tous les éléments de la classe $\boldsymbol{M}$, est une classe d'ensembles décroissants. Par conséquent, pour que la classe $M$ soit saturée (cond. 6), il faut bien que $B$ soit son élément.

Nous avons ainsi montré qu'il existe dans $\boldsymbol{M}$ un ensemble $B$ tel que

$$
b \varepsilon B \text { et } a \text { non }-\varepsilon B
$$

ce qui prouve que la condition $(\gamma)$ est aussi vérifiée.

Donc, si une classe $\boldsymbol{M}$ ordonne l'ensemble $M$ au sens donné par notre définition, la relation $n<4$, correspondante à cette classe, ordonue l'ensemble $M$ au sens ordinaire.

2. Le théorème réciproque est également vrai.

Admettons, en effet, que la relation $\varrho$ vérifie les conditions $(\alpha)-(\gamma)$ par rapport à tous les éléments de $\boldsymbol{M}$. Soit $\boldsymbol{M}$ la classe 
de tous les restes, qui correspondent à cette relation. $\boldsymbol{M}$ est évidemment une classe des sous-ensembles décroissants de $\boldsymbol{M}$. Nous montrerons, qu'elle est saturée par rapport à cette propriété.

Supposons, qu'il n'en est pas ainsi. Il existerait done un tel sous-ensemble $E$ de $M$, qui n'en serait pas un reste et qui vérifierait pour tout reste $X$ la formule:

\section{$X \subset E$ ou bien $E \subset X$.}

$E$ n'étant pas un reste de $M$, il existe dans $M$ deux éléments a et $b$ qui satisfont aux conditions:

$$
a \varepsilon E, \quad a \varrho b, \quad b \text { non }-\varepsilon E .
$$

Désignons par $R$ l'ensemble de tous les $x$, pour lesquels on a: $a \varrho x ; R$ est bien un reste de $M$; d'autre part,

tandis que

$$
a \varepsilon E \text { et anon- } \varepsilon R \text {, }
$$

$$
b \varepsilon R \text { et } b \text { non }-\varepsilon E \text {. }
$$

Donc, ni $E$ n'est contenu dans $R$, ni $R$ dans $E$ - contrairement à l'hypothèse.

Il en résulte que $\boldsymbol{M}$ est une classe saturée (cond. 6).

Nous avons ainsi montré, que la classe $\boldsymbol{M}$ de tous les restes de $M$ élablit un ordre dans $M$ (au sens de la définition I) et que cet ordre est bien identique à celui qui y établit la relation $\rho$.

$$
\text { C. Q. F. D. }
$$

Nous allons montrer à présent que $\boldsymbol{M}$ est. l'unique classe, qui y établit cet ordre.

Supposons à ce but, que la classe $\boldsymbol{N}$ jouit de la même proprieté. Je dis, que tout élément de $N$ est un reste de $M$. Soit, en effet, $A$ un élément quelconque de $N$ et $a$ un élément de $A$. Soit $x$ un élément quelconque, qui satisfait à la formule: $a \varrho x$; il s'agit de montrer que $x \varepsilon A$. Supposons le contraire; alors,

$$
a \varepsilon A \text { et } x \text { non- } \varepsilon A \text {, }
$$

ce qui prouve, que $x$ précède $a$, c'est à dire: $x \varrho a,-$ contrairement à $(\beta)$.

Donc, $x \varepsilon A$ et, par suite, $A$ est un reste de $M$. Cette conclusion peut s'écrire:

$$
N \subset M
$$


Comme, d'autre part, $\boldsymbol{N}$ est saturée (cond. 6), - on en déduít l'identité

$$
\boldsymbol{N}=\boldsymbol{M} .
$$

Nous avons ainsi mis en évidence la correspodance biunivoque entre les classes qui nétablissent l'ordreu dans un ensemble donné et les diverses façons d'ordonner cet ensemble.

Passons maintenant aux ensembles bien ordonnés.

Convenons de dire, que $\boldsymbol{M}$ est une ${ }_{n}$ classe bien ordonnée des ensembles décroissants", lorsque

(7) toute sous-classe $\boldsymbol{X}$ de $\boldsymbol{M}$ contient un tel élément que tous les autres en sont des sous-ensembles.

D'une façon analogue, on établit le sens du terme „classe bien ordonnée des ensembles croissants".

Définition II. La classe $\boldsymbol{M}$ établit un bon ordre dans l'ensemble $M$, lorsqu'elle est saturée parrapportà la propriété d'être une ${ }_{n}$ classe bien ordonné des sousensembles décroissants de $M$.

Cela signifie, que la classe $M$ vérifie les conditions 1,7 et la condition:

(8) M n'est pas une vraie sous-classe d'aucune classe satisfaisante aux conditions 1 et 7 .

Envisageons une classe $\boldsymbol{M}$ qui vérifie les conditions 1,7 et 8 . Nous montrerons, qu'elle satisfait aussi aux cond. 2 et 6.

Quant à la cond. 2, il est intéressant de voir que déjà la cond. 7 seule l'implique. En effet, soit $X$ et $Y$ deux éléments quelconques de $\boldsymbol{M}$ et soit $\boldsymbol{K}$ la classe composée de ces deux éléments. Selon (7) un d'eux est le plus grand élément de cette classe; suivant que c'est $X$ ou $Y$ on a:

\section{$Y \subset X$ ou bien $X \subset Y$}

ce qui veut dire, que la condition 2 est vérifiée ${ }^{1}$ ).

La classe $M$ est saturée par rapport aux cond. 1 et 2 . En effet, supposons, qu'on puisse ajouter à la classe $M$ un élément $E$, ne lui appartenant pas, sans que la classe $N$, ainsi obtenue, cesse vérifier les cond. 1 et $2 ; \boldsymbol{N}$ vérifierait évidemment aussi la condi-

1) Ce raisonnement est dú à M. Saks: 
tion 7, - contrairement à l'hypothèse que $\boldsymbol{M}$ est saturée (condition 8).

Donc, la classe $M$ satisfait aux conditions 2 et 6 et elle établit un ordre dans l'ensemble $M$ au sens de la définition I. Nous allons voir que cet ordre est un bon ordre.

Soit $A$ un sous-ensemble quelconque de $M$ et $A$ la classe des sous-ensembles de $A$ tels que pour chacun d'eux existe au moins un élément de $A$ qui lui appartient, et au moins un qui ne lui appartient pas. Soit $S$ le plus grand de ces ensembles. $S$ contient tous les éléments de $A$, excepté un seul. En effet, supposons que deux éléments $a$ et $b$ de $A$ n'appartiennent pas à $S$ et que $a<b$; il existerait done un ensemble $B$ tel que

\section{$b \varepsilon B$ et $a$ non $-\varepsilon B$}

or, selon la défiuition de $S, B \subset S$; donc $b \varepsilon S$; ce qui est impossible.

Il en résulte, que l'élément de $A$, qui n'appartient pas à $S$, précède tous les autres éléments de $A$; en d'autres termes: l'ensemble $M$ est bien ordonné au sens ordinarre.

D'autre part $M$ étant bien ordonné, la classe de tous ses restes est évidemment une nclasse bien ordonnée des sous ensembles décroissants de $M^{u}$ et elle est sa tu rée par rapport à cette propriété.

Ainsi, l'emploi de la définition II est complètement justifié. - Définition III. Le bon ordre établi par $M$ dans un ensemble $\boldsymbol{M}$ est une suite ${ }^{1}$ ) finie, lorsque

(9) $\boldsymbol{M}$ est une „classe bien ordounée des ensembles croissants".

Les conditions 1,7 et 9 étant vérifiées, toute sous-elásse de $\boldsymbol{M}$ contient un élément, qui y est le plus grand, et un autre, qui y est le plus petit. Pour obtenir une définition de suite infinie (du týpe $\omega$ ), on suppose que la cond. 9 n'est pas vérifiée et on la remplace par la suivante:

(10) $\boldsymbol{X}$ étant une sous-classe de $\boldsymbol{M}$ telle que la somme de ses éléments n'est pas identique à $M$, il existe un élément de $\boldsymbol{X}$, qui y est le plus petit.

Dans ma Note précitée se trouve la définition suivante de l'ensemble fini:

1) Il n'est question ici que des suites dont tous les termes sont différents. 
Définition IV. $M$ est un ensemble fini, lorsque la classe de tous ses sous-ensembles est l'unique classe, qui vérifie les conditions suivantes:

(11) tous ses. éléments sont des sous-ensembles de $M$,

(12) tous les ensembles, composés d'un seul élément de $M$, lui appartienvent,

(13) $X$ et $Y$ étant ses éléments, $X+Y$ l'est aussi.

Il est important de voir qu'on peut démontrer le théorème suivant, sans faire intervenir la notion du nombre naturel:

pour qu'un ensemble soit fini au sens dela déf. IV, il faut et il suffit, qu'il puisse être rangé en une suite finie au sens de la déf. III ${ }^{1}$ ).

Je démontre ce théorème par une méthode, qui est en harmonie complète avec la théorie des ensembles de M. Zermelo et qui ne fait d'ailleurs l'usage que des cinq premiers axiomes de cette théorie (sans avoir recours à l'axiome "du choix" et à celui ${ }_{n}$ de l'infini" ${ }^{4}$ ).

Théorème $I$. Tout ensemble composé d'un seul élément est fini.

Théorème II. $A$ et $B$ étant fini, leur somme $A+B$ est finie.

Démonstration. Soit $\boldsymbol{K}$ une classe, composée uniquement des sousensembles de $A+B$, telle que

(i) tout ensemble composé d'un seul élément de $A+B$ est un élément de $K$.

(ii) $X$ et $Y$ étant des eléments de $K$, il en est de même de $X+Y$.

Il s'ugit de montrer, que $K$ contient tous les sous-ensembles de $A+B$.

Soit $\boldsymbol{A}$ la classe de tous les sous-ensembles de $\boldsymbol{A}$ qui appartiennent à $\boldsymbol{K}$. En vertu de $(i)_{i} \boldsymbol{A}$ admet comme éléments tous les enscmblos composés d'un seul élément de $A_{\text {; }}$ en vertu de (ii), elle contient la somme de tous deux de ses éléments. Or, $\boldsymbol{A}$ étant fini, $\boldsymbol{A}$ est identique à la classe de tous les sous-ensembles de $A$; par suite, $\boldsymbol{K}$ contient tout sous-ensemble de $A . \quad 11$ en est de même de $B$. D'autre part, comme tout sous-ensemble de $A+B$ est une somme d'un sousensemble de $A$ et d'an sous-cnsemblo de $B$, - tout sous-ensemble de $A+B$ appartient à $\dot{K}$, en vertu de la cond. (ii).

Théorème III. Tout sous-ensemble d'un ensemble fini est fini.

Démonstration. La classe de tous les sous-ensembles finis d'un ensemble donné $M$ satisfait - d'après les théorèmess I et II - anx conditions (11)-(13). Or, $M$ étant fini, elle est identique à la classe de tous les sous-ensembles de $M$.

Théorème 1V. Tout ensemble qui peut être rangé en une suite finie, est fini.

Démonstration. Soit $M$ un ensemble donné et $\boldsymbol{M}$ une classe qui le range en une suité finie. Soit $\boldsymbol{K}$ la classe de tous les ensembles finis qui sont des éléments de $M$.

1) On montre aisément l'équivalence de la définition III et de celle de ${ }_{n}$ 'ensemble doublement bien ordonné ${ }^{\prime}$ de M. Zormelo (Sur las ensembles finis ot le principe de linduction complète, Acta Mathematica 1909). 
D'après la déf. III - il existe dans $M$ un élément qui y est le plus petit; celui-ci ne peut contenir plus qu'un seul élément, puisquo la classe $\boldsymbol{M}$ est saturée; par conséquent (théor. $\mathbf{I}$ ), il est un ensemble fini. Donc, la classe $\boldsymbol{K} \mathbf{n}^{\prime}$ est pas vide. Soit $K$ le plus grand de ses éléments.

Supposons - contrairement à la thèse du théorème - que $M$ n'est pas fini; c'est à dire, que la classe $\boldsymbol{M}-\boldsymbol{K}$ n'est pas vide. Soit $L$ son élément le plus petit. En vertu du théor. III, on a: $K \subset L$.

La classe $M$ étant saturée, les ensembles $L$ et $K$ ne diffèrent que par un seul élément; donc, (théor. I et II) $-K$ étant fini il en ost de même de $L$. Ainsi, notre supposition, que l'ensemble $M$ n'était pas fini, était fausse. C.Q.F. D.

Théordme $V$. 'Tout onsomble composé d'un seul élément peut être rangé en une suite finie.

Démonstration. En effet, la classe dont cet ensornblo est l'unique élément lo range on une suite finie.

Théorème $V I$. Si les ensembles $A$ et $B$ peuvent être rangés en une suite finie, leur sommo $A+B$ peut l'être aussi.

Démonstration. Soit $A$ une classe qui range l'ensemble $A$ en une suite finie et soit $B$ une clusse qui le fait par rapport is $B$. Soit $D$ la classe que l'on obtient de $A$ en $y$ remplaçant chaque élément $X$ par la somme $X+B$. Posons $\boldsymbol{C}=\boldsymbol{D}+\boldsymbol{B}$; je dis, que la classe $\boldsymbol{C}$ range l'ensemble $\boldsymbol{A}+\boldsymbol{B}$ en une suite finie.

En effet, on roit immédiatement, que $C$ satisfait aux conditions 1,2 et 6. Nous montrerons qu'elle vérifie anssi les conditions 7 et 9 . Soit $K$ une sousclasse quelconque de $\boldsymbol{C}$. Si on suppose que $\boldsymbol{K} \subset \boldsymbol{B}$, il en résulte évidemment, que $\boldsymbol{K}$ contient un élément le plus grand et un le plus petit; supposons donc que cette inclusion n'est pas vérifiée. Tout élément de $\boldsymbol{K}$ qui appartient i $\boldsymbol{D}$ peut être représenté sous la forme $X+B$, oi $X \in A$; soit $X_{1}$ le plus grand $X$ et $X_{2}$ le plus petit. Or, $X_{1}+B$ est le plus grand élément de $K$. Quant à l'élément qui y est le plus petit, deux cas pouvent se présenter:

1. $\boldsymbol{K} \subset \boldsymbol{D}$, donc $X_{1}+B$ est le plus petit élément de $\boldsymbol{K}$;

2. dans lo cas contraire, il existe dans $\boldsymbol{K}$ des éléments de $\boldsymbol{B}$; le plus petit de ces éléments est évidemment un sous-ensemble do tout autre clément de $\boldsymbol{K}$.

Donc. toute sous-classe $\boldsymbol{K}$ de $\boldsymbol{C}$ contient un élément le plus petit et un le plus grand (cond. 7 et 9). En vertu de la déf. III - cela veut dire, que $\boldsymbol{C}$ range l'ensemble $A+B$ en une suite finie.

C. Q. F. D.

Corollaire. Tout ensemble firi peut être rangé en une suite finie.

Démonstration, Soit $M$ un ensemble fini. Envisageons la classe de tous les sous-ensembles de $M$, qui peuvent être rangés en une suite finio. En vertu des théor. $\nabla$ et $V I$ et de la déf. IV - cotte classe contient tous les sous-ensembles de $M$ et, en particulier, l'ensemble $M$ lui-même.

C. Q. F. D.

Ce corollaire et le théorème IV impliquent immédiatement le théorème que nous nous avions proposé a établir:

Théorème. Pour qu'un ensemble soit fini, il faut et il suffit, qu'il puisso être rangé en une suite finie.

Nous terminons cette note par une remarque suivante sur la notion de paire ordonnée. 
Soit $A$ un ensemble composé de deux éléments $a$ et $b$. à savoir:

Il n'existe que deux classes, qui établissent un ordre dans $A$,

$$
\left.((a, b),(a))^{1}\right) \text { et }((a, b),(b)) \text {. }
$$

Il semble bien naturel d'admettre la définition suivante:

Définition V. La classe $((a, b),(a))$ est une "paire ordonnée dont $a$ est le premier élément et $b$ est le second".

La notion de paire ordonnée est, comme on sait une des plus importantes dans la théorie des ensembles et il est bien utile d'avoir pour elle une définition ${ }^{2}$ ) suffisamment simple. En admettre celle que nous venons de proposer est une conséquence immédiate de l'emploi de la théorie de l'ordre qui a été discutée ici.

J'adresse mes remerciements à $\mathrm{M}$. Knaster qui a bien voulu m'aider à rédiger cette note.

1) $((a, b),(a))$ est la classe composée de deux éléments: $1^{0}$ de l'ensemble $A$ et $2^{\circ}$ de l'ensemblo dónt $a$ est l'élément unique.

2) M. Hausdorff (l. c., p 32) introduit la notion de paire ordonnée de la manière suivante:

Soit $a$ et $b$ deux éléments quelconques; soit 1 et 2 deux éléments diffèrents, dont aucun n'est identique ni à $a$ ni à $b$; les classes

$$
((a, 1),(b, 2)) \text { et }((a, 2),(b, 1))
$$

sont des paires ordonnées.

Cette définition me semble moins commode, car les éléments 1 et 2 ne penvent pas être déterminés indépendemment de $a$ et $b$; en effet, si on leur assigne un sens indépendant de $a$ et de $b$, on doit modifier la définition pour la cas, où $a$ ou $b$ coïncide avec 1 ou 2 . 